\title{
COGNITIVE FRAMING IN ACTION
}

John M. Huhn III, Cory Adam Potts, David A. Rosenbaum

Department of Psychology

Pennsylvania State University

University Park, PA 16802 USA

Corresponding author:

John M. Huhn III

441 Moore Building

Pennsylvania State University

University Park, PA 16802 USA

JMH734@PSU.EDU

Running Head: Cognitive Framing in Action

Invited revision prepared for Cognition in consultation with

http://www.elsevier.com/journals/cognition/0010-0277/guide-for-authors

\{No abstract length limit at Cognition $\}$

Cognitive Framing in Action, Page 1 of 37

(C) 2016. This manuscript version is made available under the Elsevier user license http://www.elsevier.com/open-access/userlicense/1.0/ 


\begin{abstract}
Cognitive framing effects have been widely reported in higher-level decision-making and have been ascribed to rules of thumb for quick thinking. No such demonstrations have been reported for physical action, as far as we know, but they would be expected if cognition for physical action is fundamentally similar to cognition for higher-level decision-making. To test for such effects, we asked participants to reach for a horizontally-oriented pipe to move it from one height to another while turning the pipe 180 degrees to bring one end (the "business end") to a target on the left or right. From a physical perspective, participants could have always rotated the pipe in the same angular direction no matter which end was the business end; a given participant could have always turned the pipe clockwise or counter-clockwise. Instead, our participants turned the business end counter-clockwise for left targets and clockwise for right targets. Thus, the way the identical physical task was framed altered the way it was performed. This finding is consistent with the hypothesis that cognition for physical action is fundamentally similar to cognition for higher-level decision-making. A tantalizing possibility is that higher-level decision heuristics have roots in the control of physical action, a hypothesis that accords with embodied views of cognition.
\end{abstract}

Keywords: action, cognitive framing, heuristics, object manipulation, motor control, bimanual actions 


\section{INTRODUCTION}

One of the most exciting developments in the study of cognition has been the discovery of cognitive framing effects. A famous example relates to deciding between bets presented in two ways. In one setup, participants start with $\$ 300$ and choose between getting \$100 for sure or winning \$200 with probability .5. In that case, though the expected outcome is the same for both options (\$400), people strongly prefer the first option, the "sure thing," rather than the gamble. In the other setup, participants start with $\$ 500$ and choose between losing $\$ 100$ for sure or losing $\$ 200$ with probability .5. In that case, though the expected outcome is again the same for both options (again \$400), people strongly prefer the second option, the "gamble" rather than the sure loss. This classic outcome shows that the way a choice is framed changes what is chosen (Tversky \& Kahneman, 1986).

The many examples of cognitive framing effects that have been reported in the literature have focused on questions surrounding large-scale issues such as choosing medical treatments or deciding on business investments. To the best of our knowledge, framing effects have not yet been discovered in the context of the planning of physical actions. This fact is surprising when one considers that Kahneman (2011) argued that the heuristics captured by cognitive framing reflect "fast thinking," the kind needed to make rapid decisions in everyday life, including in the savannahs and jungles from which humans evolved. If Kahneman's argument is correct, cognitive framing should appear in physical action planning because many of the decisions faced by our evolutionary ancestors were physical in nature. Kahneman made no mention of such effects in his 
book, probably reflecting his specialization in higher-level thinking rather than perceptual-motor skills.

We were interested in whether cognitive framing effects apply to physical action. Our interest in this question arose from a longstanding interest in our laboratory in the cognitive bases of physical action planning and control. Many studies in our lab have shown that even simple physical actions, like reaching for an object or flipping an inverted glass, reflect sophisticated planning comparable to that of so-called higher cognitive processes.

In one line of work, Jax and Rosenbaum (2007) and van der Wel et al. (2007) found that reaching around a barrier to a target influenced the curvature of reaching paths that followed, even when no barrier was present. This phenomenon, called hand path priming, suggested that actions performed in the recent past influence the planning and execution of current actions.

In other work, our lab provided evidence that just as actions in the past can influence present actions, actions planned for the future can also influence present actions. One anticipatory phenomenon is the end-state comfort effect. This is the tendency to grasp objects in an initially awkward posture to end in a more comfortable manner. For example, when flipping an inverted glass to fill it with water, people tend to grasp the glass with an initially awkward, thumb-down posture to end the movement in a more comfortable, easy-to-control thumb-up posture (Rosenbaum et al., 1990). Similar effects emerge when people grasp and move an elongated object, such as a standing toilet plunger, to different heights. People tend to grasp the shaft lower when moving the plunger to a higher target, and to grasp the shaft higher when moving the plunger to a 
lower target—the so-called grasp-height effect (Cohen \& Rosenbaum, 2004). Both the end-state comfort effect and the grasp-height effect ensure that the actor ends in a comfortable, easy-to-control, mid-range arm posture. For reviews of this work and related work from our and other laboratories, see Rosenbaum et al. (2012, 2014).

Results like these show that cognition and action go hand in hand. In fact, the rich cognitive substrates that underlie physical action have led some researchers to claim that intellectual abilities are fundamentally similar to, and possibly rooted in, perceptualmotor control (Calvin, 1994; Piaget, 1954; Rosenbaum, Carlson, and Gilmore, 2001). Consideration of this possibility led to the main question in the present study: If the cognitive substrates of physical action planning and intellectual planning are fundamentally similar, then are physical actions subject to cognitive framing effects?

To pursue this question, we need to say what a cognitive framing effect is. Following Levin, Schneider, and Gait (1998), we defined a framing effect as a change in the way a task is carried out depending on how the task is presented. Said another way and focusing on the way we pursued the question at hand here- - is physical action planning subject to cognitive framing? - we defined a cognitive framing effect as one in which the same task is physically performed in different ways depending on how the task is presented. To the best of our knowledge, no such effects have been reported, though as we argue in the General Discussion section, some previously observed phenomena can be understood, in hindsight, to have reflected cognitive framing in physical action planning.

\section{EXPERIMENT 1}

The task we used entailed bimanual object manipulation. We pursued this task for two main reasons. One was that research on bimanual movements has revealed a strong 
preference for bimanual symmetry (e.g., Hughes et al., 2011; Huhn et al., 2014; Janssen et al., 2009; Kelso et al. 1979; Kelso, 1984; Kunde \& Weigelt, 2005; Marteniuk et al. 1984; Mechsner et al., 2001, 2004; Oliveira \& Ivry, 2008; Rosenbaum, Dawson, \& Challis, 2007; van der Wel \& Rosenbaum, 2010; Weigelt et al., 2006). We thought that if cognitive framing effects apply to physical actions, they would appear in bimanual movement control.

Second, very little research has been done on the manipulation of large objects requiring, or potentially requiring, use of two hands. Most of the work on bimanual control that has been done on object manipulation has focused on the manipulation of two objects with two hands (one object per hand). Studies using that task have yielded evidence for the bimanual-symmetry preference referred to above (Huhn, Schimpf, \& van der Wel, 2014; Janssen et al, 2009; Kunde \& Weigelt, 2005; van der Wel \& Rosenbaum, 2010). We were interested in what would happen when people moved a large object with two hands. We thought cognitive factors might play an important role here not just on general grounds that skilled action requires knowledge, but also on the grounds that one study has already shown that the end-state comfort effect, a cognitive planning effect for one-hand object manipulation, also holds for manipulation of large objects requiring two hands (Lam, McFee, Chua, \& Weeks, 2006).

\subsection{Method}

The setup we used is shown in Figure 1. The apparatus consisted of a PVC pipe with green tape around one end and blue tape around the other. The pipe rested in gaps between wooden boards on either side of a door, with letters above the gaps on both sides of the door. 


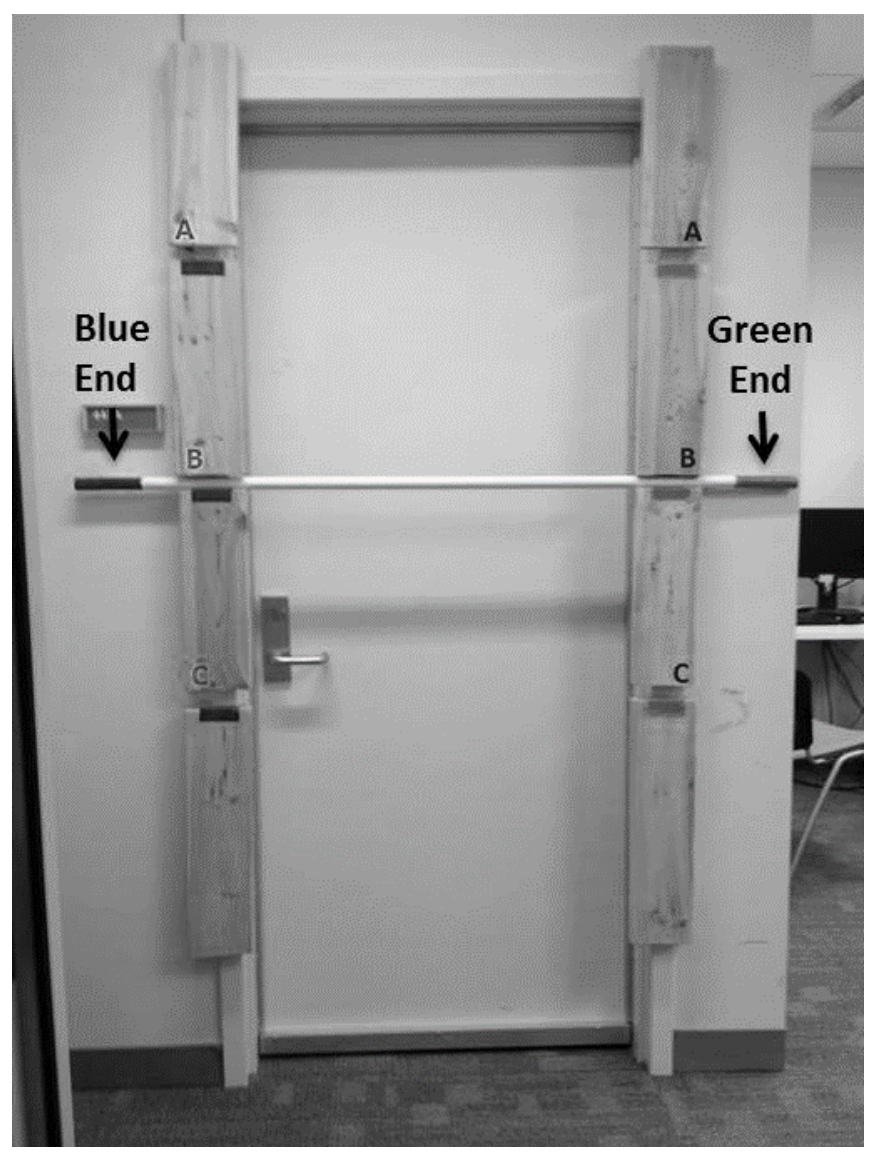

Figure 1. Gap apparatus. The plastic pipe to be moved and turned 180 degrees is in the B gaps. The pipe was brought from every height to every other height and always required a 180 degree rotation. The words "Blue End" and "Green End" were not visible to the participants. They are added here to indicate which end was blue and which end was green in this particular condition. Not visible here, all of the left targets were blue (blue tape) and all of the right targets were green (green tape).

In each trial, the participant stood facing the apparatus with his or her hands down by his or her sides, at which time the experimenter announced the task to be performed (in a manner described below). The task always involved removing the pipe from its initial height, flipping the pipe, and bringing the pipe to a new height. Because the pipe was always flipped, the end that was on the left was always brought to a target on the right and vice versa. After the participant brought the pipe to the target, s/he let go of the pipe and brought his or her hands back to his or her side in anticipation of the next trial. 
Participants were divided into two instruction groups. One group heard the color name of the pipe end that would be moved followed by the name of the target to which it would be moved (e.g., "Green end to blue A"). The other group heard the name of the target to which a pipe end would be moved followed by the color name of pipe end that would be brought there (e.g., "Blue A gets green end"). We used these two instruction orders to see whether the sequence of instructions would affect the likelihood of using one strategy or the other. We thought the order of instructions might influence the choices, consistent with a cognitive framing account. For example, calling attention to the business end of the pipe followed by the target could have primed a rotation in the angular direction of the arc from the business end to the target. Conversely, calling attention to the target followed by the business end could have primed a rotation in the angular direction of the arc from the target to the business end. Such an outcome would have been consistent with verbally mediating cognitive framing. On the other hand not finding such an outcome would not disconfirm cognitive framing; it would merely fail to provide support for verbally mediated cognitive framing.

To return to the most important point, the critical feature of our task was that from a physical perspective, every pipe transfer from one height to another could be achieved in just one way, either clockwise or counter-clockwise. If the way the pipe was transferred from a given starting height to a given target height depended on whether the left business end went to a right target or the right business end went to a left target, that would be a framing effect. 


\subsubsection{Participants}

The experiment had 48 participants (36 females; mean age 19.77 years, range 18 to 25), 24 of whom were assigned to one instruction group and the other 24 of whom were assigned to the other instruction group. The assignment of participants to instruction groups was random. All participants were volunteers from the Penn State community and none reported neurological disorders. Participants completed a short form of the Edinburgh handedness inventory (Oldfield, 1971). Of the participants, 47 reported being right-handed.

\subsubsection{Apparatus}

The gaps were spaces between wooden boards attached to the frame of a door. Each gap was $3.2 \mathrm{~cm}$ wide. The top boards were $0.39 \mathrm{~m}$ long, the four middle boards were 0.43 $\mathrm{m}$ long, and the left and right bottommost boards were $0.55 \mathrm{~m}$ long. The boards on the left were $0.82 \mathrm{~m}$ from the boards on the right. The top gap was centered at $1.80 \mathrm{~m}$ above the floor, the middle gap was centered at $1.34 \mathrm{~m}$ above the floor, and the bottom gap was centered at $0.88 \mathrm{~m}$ above the floor. The three target gaps on the left were marked with blue tape and were referred to as "blue A," "blue B," and "blue C." The three target gaps on the right side were labeled with green tape and were referred to as "green A," "green B," and "green C."

The PVC pipe was $1.52 \mathrm{~m}$ long, $0.02 \mathrm{~m}$ in diameter, and weighed 491 grams. One end of the pipe was marked with a strip of $0.05 \mathrm{~m}$ green duct tape and the other was marked with a 0.05 strip of blue duct tape.

Three webcams (Logitech QuickCam Ultra Vision, Model Number: V-UBH44) captured the movements of each participant. One webcam was placed to look at the front 
of the participant, a second was placed to look at the side of the participant, and a third was placed to look at the back of the participant. The webcams recorded participants' movements during each trial. Using the Avidemux video-analysis program (http://fixounet.free.fr/avidemux/), our research assistants viewed the video records frame-by-frame and coded the actions of interest with respect to direction of rotation. We analyzed these data to obtain the means of the rotation directions for the various conditions per subject and performed statistical analyses on these means. We used this data analysis process for all of the experiments presented here.

\subsubsection{Procedure}

At the start of each trial, the PVC pipe was positioned at one of the three heights $(1.80$ $\mathrm{m}, 1.34 \mathrm{~m}$, or $0.88 \mathrm{~m})$. In each trial, the participant's task was to listen to an instruction read aloud by the experimenter and then, using the grasp orientation(s) of his or her choice, to move the designated end of the pipe to the designated target. The instructions referred to the colored end of the pipe followed by the colored target gaps at height A, B or $\mathrm{C}$, or the same elements presented in the opposite order.

The 24 participants in each of the instruction-order groups completed 24 trials (2 possible pipe orientations at each starting position $\times 2$ possible orientations at each target position $\times 3$ target heights $\times 2$ color specifications) arranged in a Latin square design. Trials were arranged in pseudo-random order but with the stipulation that the ending position of the pipe in each trial was the starting position for the following trial. The starting position of the pipe was balanced over subjects. As said earlier, the pipe was always raised or lowered and was always rotated. The pipe's starting position in any given trial was the position to which it was brought at the end of the previous trial. In 
those rare cases where the pipe was brought to an incorrect position (less than $2 \%$ of the trials, never more than once for any subject, and always in early trials), the experimenter repositioned the pipe at the start position for that trial after telling the subject to turn around so s/he could not see how the experimenter handled the pipe, lest his or her behavior bias the subject's own performance. After the experimenter repositioned the pipe and said "OK," the subject turned around, waited a moment, as per prior instruction, and then completed the trial again. The number of such error trials was very small. When all 24 trials were complete, the participant was debriefed. This same procedure with respect to errors was used in the studies that follow.

\subsection{Results}

We analyzed the mean frequencies of clockwise rotations with an ANOVA designed to evaluate the effect of target direction (left or right) $\times$ target height $(1.80 \mathrm{~m}$ or $1.34 \mathrm{~m}$ or $0.88 \mathrm{~m}) \times$ instruction group (pipe-location or location-pipe). The ANOVA yielded a significant main effect of target direction, $\mathrm{F}(1,46)=37.85, p<.001, \eta^{2}=.45$, such that when the target was on the left the frequency of clockwise rotations was low $(M=0.31$, $S E=.05)$, whereas when the target was on the right the frequency of clockwise rotations was high $(M=.70, S E=.05)$. There were no other main effects or interactions; all $p$ 's $>$ $.05 .^{1}$

Regarding the effects of instruction order, as implied by the last statement, there was not a main effect of this independent variable. The associated $p$ value was $p=.67$. Nor was

\footnotetext{
${ }^{1}$ Note that the ANOVA design was functionally equivalent to looking at start direction $\times$ start height or, for that matter, start direction $\times$ target height, or target direction $\times$ start height. In other words, start direction and target direction were perfectly confounded, so we could not statistically isolate the effects of start heights versus target heights. We were aware of this going into the experiment but were not concerned about it because of the vast amount of evidence indicating that target positions matter much more than start positions in the way objects are grasped and moved to target destinations. For a review, see Rosenbaum et al. (2012).
} 
there an interaction between instruction order and target direction or an interaction between instruction order and target height. The associated $p$ values were $p=.23$ and $p=$ .85 , respectively.

At the end of each participant's testing session, both in the present experiment and in the two experiments to follow, we asked participants to tell us what strategy, if any, they used. The majority of participants admitted that they did not put much thought into the way they moved the pipe. Instead, they said they mostly paid attention to what the business end was (our term, not theirs) and where that end of the pipe was supposed to go. When asked about how they moved the pipe and what strategy they used, most of our participants said they were unaware that they had shown any specific preferences.

\subsection{Discussion}

Experiment 1 shows that when participants were told to bring a specified pipe end (the "business end" of the pipe) to a target on the right, they preferred clockwise rotations, but when the same participants were told to bring the business end of the pipe to a target on the left, they preferred counter-clockwise rotations. Another way of saying this is that participants tended to bring the business end of the pipe through the upper part of the workspace (i.e., above the pipe's axis of rotation). Relying on this more concise way of characterizing the tendency, we call this the upper rotation effect. Because the upper rotation effect reflected the way the instructions were given, albeit without regard to whether the business end or the target location was named first, we view the effect as a cognitive framing effect. To the best of our knowledge, this is the first demonstration of a cognitive framing effect in physical action. 


\section{EXPERIMENT 2}

A way to test the hypothesis that the upper rotation effect stems from cognitive factors is to ask whether the effect is eliminated when there was no business end. We addressed this possibility in Experiment 2 by not mentioning a business end and by not mentioning a target side. In Experiment 2, we told our participants that they would always rotate the pipe 180 degrees and that they would always move the pipe (with rotation) from whatever height it occupied at the start of the trial to whatever level was named by the experimenter. For example, the pipe might be in the B gaps and the experimenter would say simply, "A," whereupon the subject would remove the pipe from B, turn it and then raise it to insert it into the A gaps with a 180 degree pipe rotation.

The prediction was straightforward: If the upper rotation effect in Experiment 1 was a cognitive framing effect and if, moreover, the upper rotation effect counteracted an underlying tendency for a given subject always (or mostly) to turn the pipe clockwise or counter-clockwise, then individual subjects in Experiment 2 would always turn the pipe one way or the other. A given subject, in other words, would always (or nearly always) turn the pipe clockwise or counterclockwise. Adopting that strategy would depart markedly from what we observed in Experiment 1, where subjects switched between clockwise and counterclockwise rotations depending on the instruction about which end of the pipe was the business end and which side of the door frame was the target.

\subsection{Method}

\subsubsection{Participants}

Twenty-four volunteers from the Penn State community (23 female; mean age 19.17, range 18-29) participated. No subject reported any neurological disorder. Participants 
completed a short form of the Edinburgh handedness inventory (Oldfield, 1971). Of the participants, 23 reported being right-handed.

\subsubsection{Design and Apparatus}

The apparatus was the same as in Experiment 1.

\subsubsection{Procedure}

Participants followed a procedure similar to the one in the previous study. They were told that they were always required to rotate the pipe. In each trial, participants listened to an instruction from the experimenter that referred to the height of the next target. For the three target heights of $1.80 \mathrm{~m}, 1.34 \mathrm{~m}$, and $0.88 \mathrm{~m}$, the instruction from the experimenter was simply "A," "B," or "C," respectively. There was no mention of the colored end of the pipe or the colored target gaps. Participants were told that the colored ends and the marked target gaps were being used for another study.

All 24 participants experienced 24 trials $(2$ starting orientations $\times 3$ target heights $\times 2$ possible targets for each trial (the two heights not currently occupied by the pipe) $\times 2$ trials per condition).

\subsection{Results}

We needed a way of analyzing the data that would be commensurate with the way we analyzed the data in Experiment 1, but because there was no business end in Experiment 2, there was no way to say whether participants intended to direct the left end of the pipe to the right target side or vice versa (if indeed they had either of those intentions). To address this data-analysis challenge, we looked at the probability of turning the pipe clockwise in Experiment 2, and looking back at the Experiment 1 data, we removed target side (or business-end side) as a factor, so we could compare the probability of 
turning the pipe clockwise in Experiment 1 with the probability of turning the pipe clockwise in Experiment 2. We reasoning that if the statistical distribution of clockwise rotations was different in Experiments 1 and 2, and in particular if the prevalence of a single rotation direction per subject was higher in Experiment 2 than in Experiment 1, then that result would accord with the hypothesis that the upper rotation effect in Experiment 1 counteracted a tendency to turn the pipe in a consistent direction per participant—either always (or mostly) clockwise, or always (or mostly) counterclockwise.

In Figure 2 we show the number of participants in Experiments 1 and 2 who showed every possible number of clockwise rotations out of the maximum possible number of 24 per participant. As seen in the left panel, the peak of the distribution was at 12 (an equal split of clockwise and counter-clockwise rotations) for Experiment 1, whereas the distribution was U-shaped for Experiment 2, indicating that subjects in Experiment 2 were likely either to use a clockwise or a counterclockwise rotation direction. 

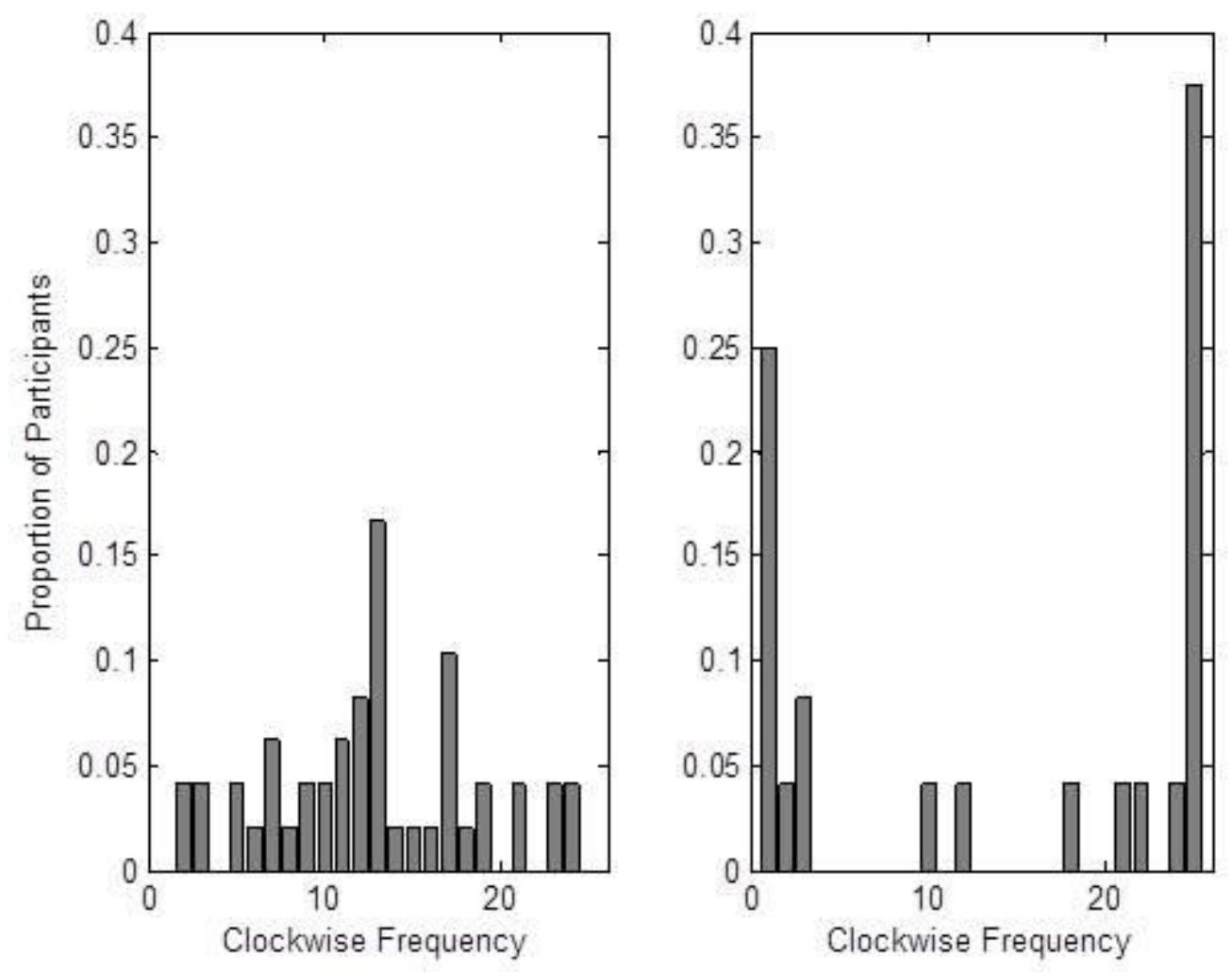

Figure 2. Frequency of clockwise rotation in Experiment 1 (left panel) and Experiment 2 (right panel). Experiment 1 had 48 participants and Experiment 2 had 24 participants.

To test the difference between these two distributions, we derived a dominant-strategy index per participant. The index was defined as the proportion of all turns per subject that went in that subject's preferred turn direction. If a subject made all turns in the clockwise or counter-clockwise direction, his or her dominant-strategy index was 1. If a subject made an equal number of turns in the clockwise and counter-clockwise direction (no preference), then his or her dominant-strategy index was .5. We submitted the observed values, which could lie between .5 and 1 , to independent-samples $t$-tests of the dominantstrategy means for Experiments 1 and 2. Via this test, we confirmed that the dominantstrategy index was strong in Experiment $2(M=.93, S E=.03)$ but was weaker in 
Experiment $1(M=.70, S E=.02)$. The null hypothesis of a zero difference between the means in the two experiments could be rejected $(p<.001)$.

\subsection{Discussion}

The difference between the results of Experiments 1 and 2 highlights the importance of the explicit information that was presented to subjects in the first experiment. When participants in Experiment 1 heard what the business end and target were, they imposed their own cognitive constraint on the task, and that led to their manifestation of the upper rotation effect. Our confidence in this statement was boosted by the results of the second experiment, where participants heard what the target height was but were not told what the business end was (or that there was one). In that case, they simplified their decisionmaking by always (or mostly) turning the pipe in a given direction per participant. So while subjects in Experiment 1 switched their rotation directions depending on which end of the pipe was the business end, subjects in Experiment 2 consistently turned the pipe in single angular directions.

It is important to note that we cannot say whether subjects in Experiment 2 did not show the upper rotation effect. They may have, expressing the effect insofar as some subjects might have decided always (or mostly) to move the left end of the pipe to the right target, therefore turning the pipe clockwise, whereas other subjects might have decided always (or mostly) to move the right end of the pipe to the left target, therefore turning the pipe counter-clockwise. When we debriefed the subjects in Experiment 2, our conversations failed to turn up any reliable indications that subjects were aware of this strategy. Further work using eye-movement recordings could explore the possibility that 
subjects consistently looked at the landmark sites for the experiment in a way suggestive of their use of a business-end-to-target strategy (Hayhoe, 2000; Land \& Hayhoe, 2001).

\section{EXPERIMENT 3}

In Experiment 3 we asked whether the upper rotation effect was tied to language or, instead, was tied to visuo-spatial attention. This question was worth asking even though we did not find evidence of an instruction-order effect in Experiment 1 . The absence of an effect of verbal instruction order — business end followed by target or vice versaneed not imply that verbal coding was irrelevant to the appearance of the upper rotation effect.

We addressed this question in Experiment 3 by omitting verbal instructions and using nonverbal cues. As shown in Figure 3, we used a computer monitor to show the participants the final position to which they would move the pipe given its current position. To know what the business end was while avoiding verbal instructions, we put colored tape on just one side of the pipe. In each trial, we presented a picture on a computer monitor displaying the new pipe position (i.e., the height and orientation to which the pipe would have to be brought from its current position). As before, in each trial the pipe had to be moved to a new height and had to be turned 180 degrees. We reasoned that if the upper rotation effect was tied to visuo-spatial attention without the need for (overt) linguistic input, then subjects would move the colored tape through the upper part of the visual field. Conversely, if the upper rotation effect was tied specifically to language (or verbal instructions), then subjects would not move the colored tape consistently through the upper part of the visual field. 


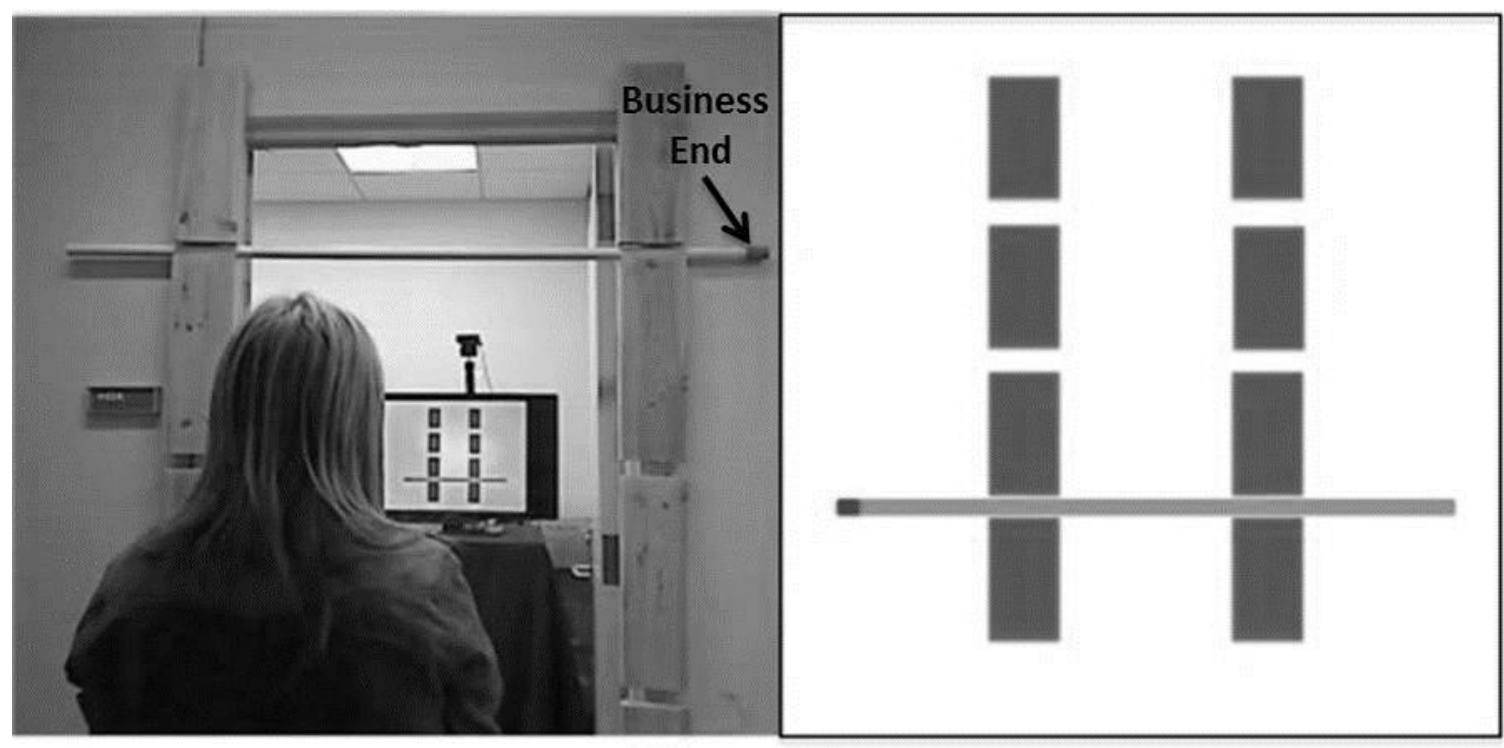

Figure 3. Setup in Experiment 3. The gap apparatus with the monitor displaying the trial's target orientation are shown in the left panel, while the stimulus appearing on the screen is shown in the right panel. As in Experiments 1 and 2, the subjects stood while performing the task. The setup shown here was the one used for half the subjects, those for whom the colored tape was at the end of the pipe. Other subjects had the colored tape near the middle of the pipe. The setups for the two groups of subjects are shown in the next figure.

Another question in Experiment 3 concerned the visual appearance of the apparatus. Did the upper rotation effect depend on the location of the business end along the pipe? In both of the previous experiments, the blue and green tapes were on the distal ends of the pipe. We wondered whether this was important. Did subjects want to see a big sweeping arc through the upper part of the workspace for some reason yet to be determined? As shown in Figure 4, we addressed this question in the third experiment by assigning participants at random to two groups based on the location of the business end. For the outer group, the business end was located as in Experiments 1 and 2, at the end of the pipe. For the inner group, the business end was brought in but not fully centered (17 $\mathrm{cm}$ away from the center). We reasoned that if the upper rotation effect depended on 
wanting to see a big sweeping arc, the upper rotation effect would be reduced or even eliminated for the inner group.

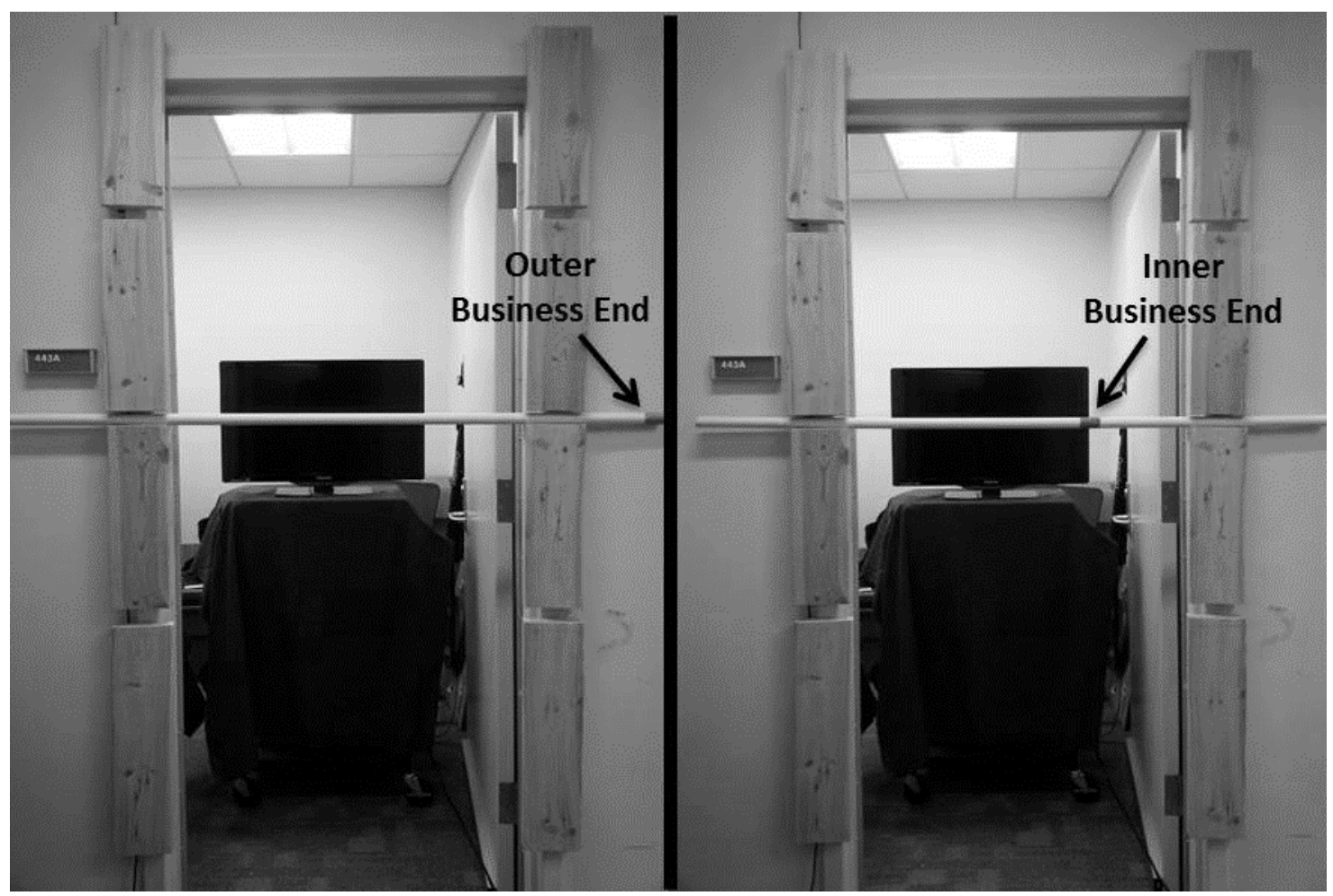

Figure 4. Setup for the outer pipe group (left) and inner pipe group (right) in Experiment 3 . In these pictures, the colored strip was green. Half the subjects in the outer group and half the subjects in the inner group had green tapes. The remaining subjects in both groups had blue tapes (not shown here). The screen is off in these pictures.

\subsection{Method}

\subsubsection{Participants}

The experiment had 24 participants ( 23 females; mean age 18.42, range 18-20). All participants were volunteers from the Penn State community and none reported any neurological disorders. Participants completed a short form of the Edinburgh handedness inventory (Oldfield, 1971). Twenty-two participants reported being right-handed. Participants were divided into two groups: the inner pipe group (6 participants each for 
the green marked pipe and the blue marked pipe), and the outer pipe group (6 participants each for the green marked pipe and the blue marked pipe).

\subsubsection{Design and Apparatus}

The gap apparatus used in the previous experiments was used in Experiment 3, except that the wooden boards were stripped of the letters and colors used to identify the targets. Regarding the PVC pipe, whereas in Experiments 1 and 2, there was green tape on one end of the pipe and blue tape on the other end of the pipe, for this study, we removed one of the tapes. For the outer group, half the subjects had the blue tape at one end of the pipe and half the subjects had green tape at one end. Similarly, for the inner group, half the subjects had blue tape near the middle of the pipe $(17 \mathrm{~cm}$ from the middle) and half of the subjects had green tape near the middle of the pipe (17 $\mathrm{cm}$ from the middle).

As shown in Figure 4, inside the doorframe to which the gap apparatus was attached was a large computer monitor (32-in. Philips Model 32PFL4507/F7, Koninklijke Philips N.V., Amsterdam, The Netherlands). The monitor was $0.61 \mathrm{~m}$ away from the outer edge of the door frame (the edge closer to the subject). The visual cues were designed in Microsoft PowerPoint and were advanced in each trial by a research assistant using a presentation clicker (Logitech, LOG910001354).

\subsubsection{Procedure}

In each trial, participants faced the apparatus. The pipe, meanwhile, lay in the apparatus. At the start of each trial, a grey screen appeared on the monitor, signaling the start of a new trial. Next, the monitor displayed the target position to which the pipe should be brought. After scanning the display the participant proceeded to move the pipe. 
There was never any mention of a business end of the pipe, and nothing was said about the colored tape.

When the PVC pipe was inserted into the gaps, the trial ended and the participant was asked to return his or her hands to his or her sides to await the next specification. On those rare occasions when subjects moved the pipe to the wrong height or failed to rotate the height (less than 2\% of the trials, never more than once for any subject, and always in early trials), the subject was asked to turn around and the pipe was repositioned by the experimenter, as in the earlier experiments.

All participants went through 24 trials ( 2 possible pipe orientations at each starting position $\times 2$ possible orientations at each target position $\times 3$ target heights $\times 2$ trials per condition).

\subsection{Results}

Even though there was no explicit mention of a business end, we defined it as the end on whose side the tape was located. The analyses we did concerned the rotation of that end. We subjected the mean frequencies of clockwise rotations to a repeated-measures ANOVA whose design was 2 (target direction, left side or right side) x 3 (target height, $1.80 \mathrm{~m}$ or $1.34 \mathrm{~m}$ or $0.88 \mathrm{~m}$ ) x 2 (tape location, inner or outer). The ANOVA revealed a significant main effect of target direction $\mathrm{F}(1,22)=832.34, p<.001, \eta^{2}=.97$. There was a strong preference to turn the pipe clockwise when the business end was brought to the right $(M=95, S E=.02)$, as compared to when the business end was brought to the left $(M=.04, S E=.02)$. There was no effect of tape location (inner or outer), $p>.05$. 


\subsection{Discussion}

In Experiment 3, we asked whether the upper rotation effect depended on the presence of verbal instructions. Our results suggested that it did not, as we still found the effect when no verbal instructions were given in the individual trials. Visual information alone drove the effect. This outcome suggests that the upper rotation effect is mainly a visuospatial phenomenon. That said, it was not a visuo-spatial phenomenon whose magnitude depended on the appearance of a big sweeping arc of the business end. The upper rotation effect was just as strong when the colored tape was close to the middle of the pipe as when the colored tape was at the end of the pipe. Participants were generally unaware of the strategy they used to turn the pipe, at least as indicated by their remarks during debriefing.

\section{GENERAL DISCUSSION}

In the three experiments reported here, we asked whether cognitive framing applies to physical action planning much as it does to higher-level decision-making. Emulating previous work on higher-level framing, we asked whether participants, given objectively equivalent tasks, would carry out the tasks differently depending on how the tasks were presented. We found that they did, as revealed by a new phenomenon reported here, the upper rotation effect, which we define as the tendency to rotate an object in such a way that the end of the object to which attention is drawn moves through the upper visual field.

In the remainder of this General Discussion section, we consider the relation of the upper rotation effect to previous findings from the field of stimulus-response (S-R) compatibility, other implications of our discovery, and remaining questions. 


\subsection{S-R Compatibility: The Top-To-Target Heuristic}

As just stated, we think our results suggest that the upper rotation effect is related to the shifting of visuo-spatial attention. It is important to consider alternative interpretations. One is suggested by research on S-R compatibility indicating that there is a natural mapping between direction of lateral motion (leftward or rightward) and direction of rotary motion (clockwise or counter-clockwise, respectively). According to an alternative interpretation suggested by this result from S-R compatibility research, the upper rotation effect mirrored a principle that has already been identified in the literature on S-R compatibility, namely, that there are natural mappings between stimuli on the left and right and rotary responses to those stimuli.

The previous work on S-R compatibility effects used reaction times. Both of the studies (Guiard, 1983; Murchison and Proctor, 2013) were prompted by the observation that when one uses a steering wheel, it is common to refer to the direction of wheel rotation in terms of the direction of motion of the top of the wheel. Interestingly, when a steering wheel is "turned to the right" the bottom is actually moved to the left, and vice versa when a steering wheel is "turned to the left." The top of the wheel carries the day, so to speak, in terms of how the lateral motion is mentally represented.

Guiard (1983) had participants rotate a wheel either clockwise or counter-clockwise in response to lights shown on the left or right. He told participants in one condition to turn the wheel clockwise when the stimulus appeared on the right, or to turn the wheel counter-clockwise when the stimulus appeared on the left. Participants were quicker to initiate the wheel motions in this condition than when the opposite instruction was given. The outcome was consistent with the hypothesis that there is a natural association 
between left and counter-clockwise rotation and between right and clockwise rotation. The same conclusion was reached by Murchison and Proctor (2013) using a similar method.

What should one make of the fact that reaction-time experiments involving quick wheel displacements yielded a principle that accords with the upper rotation effect? Because the wheel rotations that subjects made in the experiments of Guiard (1983) and Murchison and Proctor (2013) covered negligible angular amplitudes, the similarity of our results to theirs has led us to draw an important inference about our upper rotation effect, namely, that our effect had less to do with online visual control than with the application of a heuristic relating stimuli to responses. In our experiments, on-line visual control was important for bringing the pipe to the target, so it was possible that that aspect of the task was responsible for the upper rotation effect. But the fact that analogous S-R compatibility effects were found when no aiming was required - when the responses were impulses applied one way or the other-suggests that our phenomenon was not due to aiming.

Our finding may in turn inform the previous S-R compatibility work. Saying that stimuli on the left are naturally mapped to counter-clockwise turns and stimuli on the right are naturally mapped to clockwise turns is just a re-description of the results. Based on the upper rotation effect, we can propose a mechanistic account of the S-R finding. Suppose that the way a steering wheel is turned is mentally coded in terms of the lateral displacement of the top of the wheel. Suppose in addition that there is a top-to-target heuristic, a term we introduce here. The existence of this heuristic could explain why it is common to refer to the direction of wheel rotation in terms of the direction of motion of 
the top of the wheel. Further justifying this hypothesis, visual attention proceeds from top to bottom (e.g., Aaltonen, Hyrskykari, \& Räihä, 1998). In addition, while there are many languages that are read from top to bottom, we know of no language that is read from bottom to top. So the top is special.

By invoking the top-to-target heuristic, we can explain the upper rotation effect as well as the greater speed with which subjects turn steering wheels counter-clockwise to the left and clockwise to the right. A simple coding principle captures the results in both domains, bearing out the general principle, championed by Proctor and his colleagues, that stimulus and response ensembles for current tasks are cognitively coded in a way that fundamentally shapes the speed and accuracy of the observed stimulus-response translations (Proctor \& Reeve, 1990; Proctor \& Vu, 2006). ${ }^{2}$

\subsection{Other Implications}

We now turn to other implications of this study, starting with the fact that we have just offered an explanation of the upper rotation effect that joins two largely disconnected lines of lines of research: S-R compatibility research and action-planning research. Researchers in both lines have been interested in each other's work. Still, the two lines have been more separated than one might expect. The separation might be ascribed to the balkanization of research in general, but there may be another, deeper reason: The implicit rules that subjects bring to bear when they perform in S-R compatibility experiments might not correspond to the implicit rules they bring to bear in ecological contexts. There might be a narrowing of action possibilities in S-R compatibility tasks

\footnotetext{
${ }^{2}$ We refer to the phenomenon we discovered here as the upper rotation effect rather than the top-to-target effect because the former phrase echoes the observation we made, whereas the latter phrase assumes the interpretation we have given to it.
} 
that does not correspond to the widening of action possibilities in everyday life. That narrowing may be due to, or exacerbated by, the emphasis on responding as quickly as possible, which in everyday life, seldom occurs.

In the experiments reported here we did not urge our subjects to move as quickly as they could. In fact, we never placed a temporal demand on our participants. Nevertheless, the response alternatives that our participants had, at least with respect to direction of pipe rotation, were limited to just two possibilities, as in turning a steering wheel. Given this similarity to the steering-wheel studies, we believe the empirical phenomenon we have discovered is essentially the same as the one discovered by Guiard (1983) and Murchison and Proctor (2013). To the extent this is correct, an exciting outcome of the present study was that, in our attempt to understand our action-planning phenomenon, we were led to a reinterpretation of a well-known phenomenon of S-R compatibility. This convergence hints at the validity of the general principle we have suggested.

Another implication of our work pertains to the generality of cognitive framing effects. As noted in the introduction, cognitive framing effects have been studied in connection with higher-level decision-making rather than physical action planning. But if cognitive framing effects hold in the task of grabbing and rotating a pipe, they are likely to hold in other physical tasks as well where they have not been proclaimed as such. We would argue now that S-R compatibility effects may be viewed as cognitive framing effects insofar as the same response is cued in different ways by stimuli that are defined, usually ex post facto, as either more or less natural. The dependent variable in S-R studies is reaction time rather than the response that is selected, but that distinction may be artificial. How long it takes to make a response depends on how the response is 
performed physically, not just on how long subjects decide which response to make (Abrams \& Balota, 1991).

At least one other study has shown that the way responses are made depends on how they are cued. Franz and McCormick (2010) asked participants to reach to two circular targets, one corresponding to the left hand and one corresponding to the right hand. The investigators varied the instructions. In some conditions, participants were instructed to move both hands to their respective targets, while in other conditions, participants were instructed to move one hand and the other to their respective targets. Participants were significantly faster to initiate their actions when they were told to move both hands to the targets, rather than one hand and the other. The authors took this to suggest that in the "both" conditions, participants represented the task as a single bimanual movement, whereas in the "and" conditions, participants represented the task as two separate, unimanual movements. The authors did not refer to this finding as a cognitive framing effect per se but we think it can be viewed as one, albeit one in which the effect was more subtle than our upper rotation effect. Franz and McCormick's study and ours are the only two we know of where the identical physical task is cued in different ways. There are many studies where different physical tasks are asked for, of course, and those studies have shown strong cognitive effects, as in the earlier work on the end-state comfort effect and the grasp-height effect, where the same object was grasped differently depending on what was to be done with it next. But the existence of those strong cognitive effects needn't be viewed as cognitive framing effects per se because the acts to be performed were themselves different. Franz and McCormick showed first, and we have now shown second, that cognitive framing applies to physical action given the rigorous criterion of 
looking for differences in the way the same physical task is performance depending on how it is cued.

\subsection{Remaining Questions}

Questions remain about the upper rotation effect. One is whether, in our pipe transfer tasks, it actually was less computationally demanding for participants to show the upper rotation effect than to show consistent rotation directions. If participants brought the business end to its target before they brought the other end to its target in the next trial, it might have been optimal for them to approach the target with the business end from the same direction as often as possible, either from above or from below. Switching the approach direction might have required a change in the detailed aspects of the placement of the pipe's business end into the target, and might have required a change in the detailed aspects of the placement of the pipe's non-business end into the other target. If due to attention, the business end was inserted first—an assumption that says nothing about lowering versus raising the business end on the way to the first target—our participants would have actually needed to change their pipe placement behaviors for the first and second targets if they always turned the pipe clockwise or counter-clockwise.

From this perspective, it may have been less computationally demanding for participants to show the upper rotation effect than to show consistent rotation directions, always turning the pipe clockwise or always turning the pipe counter-clockwise per subject. ${ }^{3}$

\footnotetext{
${ }^{3}$ The idea that heuristics are less computationally demanding has been advocated by Griffiths \& Tenenbaum (2006). One of our participants, who was a mechanical engineering student, always turned the pipe in the same rotary direction and announced, when the task was presented to him, that it was obvious to him that this was what he should do. During debriefing, he told us that to turn the pipe the same way every time, he redefined the goal end (not his term) and the target, ensuring that the goal end would always be on the same side and the target would always be on the other side. This extra cognitive step was not needed if the goal end always went through the upper field.
} 
A second question gets back to the origins of cognitive framing effects. Are we suggesting that because we found cognitive framing effects in a simple physical-action task that cognitive framing effects trace back to physical action planning per se, perhaps as embodied by our ancient ancestors? That suggestion would fit with the tenor of Kahneman's view of quick thinking, with which we are sympathetic. We find his view appealing, especially because in other work from our lab, it has been shown that the endstate comfort effect, discussed earlier in this article, appears in nonhuman primates such as cotton-top tamarin monkeys (Weiss, Wark, \& Rosenbaum, 2007) and even in nonprimates such as lemurs (Chapman, Weiss, \& Rosenbaum, 2010). The ancestors of cotton-top tamarins split off from the hominin line 40 million years ago, whereas the ancestors of lemurs split off from the hominin line even longer ago than that, 65 million years ago (Rosenbaum et al., 2014). These results suggest that the cognitive machinery enabling action-based planning subserving the end-state comfort effect was in place at least 65 million years ago. It does not necessarily follow from this that the guiding constraint we have identified here, the top-to-target constraint, was in place that long ago. All we can say is that it might have been. If it was, or if other comparable preferences were in place long ago, then one would have a basis for tracing cognitive framing effects back to quick thinking, as Kahneman suggested. Indeed, cognitive framing effects may have originated in physical action planning. That is not a proposal Kahneman made explicitly, but we suspect it is one he would be happy to abide.

These points having been made, a word of caution is in order. Our participants were mainly university students, the sort of people who reliably show intellectually expressed cognitive framing effects. It is possible, therefore, that they showed a physically 
expressed cognitive framing effect because of their intellectual capabilities. We cannot be sure, therefore, that we tapped into a truly primitive mechanism that set the evolutionary stage for intellectually expressed cognitive framing. Alternatively, our subjects may have brought their intellectual proclivities to bear in a way that happened to be physically expressed. Our results are merely consistent with the former hypothesis. On the other hand, our results are also, and more interestingly, consistent with the thinking of Piaget (1954), who argued for tight developmental links between cognition and action, and with the thinking of Schmidt and Bjork (1992), who argued that perceptual-motor skilllearning and intellectual learning are more similar than different. That view was echoed by Rosenbaum, Carlson, and Gilmore (2001). Finally, our results fit with the findings of those who have endorsed an embodied view of cognition (Barsalou, 2008; Beilock, 2009; Glenberg \& Kaschak, 2002). They have endorsed the idea that the way we perceive and think about events is strongly mediated by physical action tendencies. To this view we have added the complementary notion that the processes used to find solutions in intellectual spheres and perceptual-motor spheres are intimately related and, potentially, the same. 


\section{AUTHOR NOTES}

Based on an unpublished masters thesis by the first author. Helpful suggestions were provided by Liana Brown, Lisa Fournier, Matt Heath, Oliver Herbort, Frank Hillary, Jim Lackner, Greg Murphy, Kristina Neely, Jeroen Smeets, Robrecht van der Wel, Dan Weiss, Brad Wyble, and anonymous reviewers. This material is based upon work supported by the National Science Foundation Graduate Research Fellowship Program under Grant No. DGE1255832 to JMH and a John Simon Guggenheim Memorial Foundation Fellowship to DAR. Any opinions, findings, and conclusions or recommendations expressed in this material are those of the author(s) and do not necessarily reflect the views of the National Science Foundation.

Correspondence should be directed to John M. Huhn III at jmh734@psu.edu. 


\section{REFERENCES}

Aaltonen, A., Hyrskykari, A., \& Räihä, K. J. (1998). 101 spots, or how do users read menus?. In Proceedings of the SIGCHI conference on human factors in computing systems (pp. 132-139). ACM Press/Addison-Wesley Publishing Company.

Abrams, R. A., \& Balota, D. A. (1991). Mental chronometry: Beyond reaction time. Psychological Science, 2, 153-157.

Barsalou, L. W. (2008). Grounded cognition. Annual Review of Psychology, 59, 617-624.

Beilock, S. L. (2009). Grounding cognition in action: expertise, comprehension, and judgment. Progress in Brain Research, 174, 3-11.

Calvin, W. H. (1994). The emergence of intelligence. Scientific American, 271, 101-107.

Chapman, K. M., Weiss, D. J. \& Rosenbaum, D. A. (2010). Evolutionary roots of motor planning: The end-state comfort effect in lemurs (Lemur catta, Eulemur mongoz, Eulemur coronatus, Eulemur collaris, Hapalemur griseus, and Varecia rubra). Journal of Comparative Psychology, 124, 229-232.

Cohen, R. G. \& Rosenbaum, D. A. (2004). Where objects are grasped reveals how grasps are planned: Generation and recall of motor plans. Experimental Brain Research, 157, 486-495.

Franz, E. A., \& McCormick, R. (2010). Conceptual unifying constraints override sensorimotor interference during anticipatory control of bimanual actions. Experimental Brain Research, 205, 273-282.

Glenberg, A. M., \& Kaschak, M. P. (2002). Grounding language in action. Psychonomic Bulletin \& Review, 9, 558-565. 
Griffiths, T. L., \& Tenenbaum, J. B. (2006). Optimal predictions in everyday cognition. Psychological Science, 17, 767-773.

Guiard, Y. (1983). The lateral coding of rotations: A study of the Simon effect with wheel-rotation responses. Journal of Motor Behavior, 15, 331-342.

Hayhoe, M. (2000). Vision using routines: A functional account of vision. Visual Cognition, 7, 43-64.

Hughes, C. M., Haddad, J. M., Franz, E. A., Zelaznik, H. N., \& Ryu, J. H. (2011). Physically coupling two objects in a bimanual task alters kinematics but not end-state comfort. Experimental Brain Research, 211, 219-229.

Huhn III, J. M., Schimpf, K. A., \& van der Wel, R. P. (2014). Symmetries in action: On the interactive nature of planning constraints for bimanual object manipulation. Experimental Brain Research, 232, 3919-3927.

Janssen, L., Beuting, M., Meulenbroek, R., \& Steenbergen, B. (2009). Combined effects of planning and execution constraints on bimanual task performance. Experimental Brain Research, 192, 61-73.

Jax, S. A. \& Rosenbaum, D. A. (2007). Hand path priming in manual obstacle avoidance: Evidence that the dorsal stream does not only control visually guided actions in real time. Journal of Experimental Psychology: Human Perception and Performance, 33, $425-441$.

Kahneman, D. (2011). Thinking, fast and slow. New York, NY: Farrar, Straus and Giroux. 
Kelso, J.A.S. (1984). Phase transitions and critical behavior in human bimanual coordination. American Journal of Physiology: Regulatory, Integrative and Comparative Physiology, 246, R1000-R1004.

Kelso, J. A. S., Southard, D. L, \& Goodman, D. (1979). On the nature of human interlimb coordination. Science, 203, 1029-1031.

Kunde, W. \& Weigelt, M. (2005). Goal congruency in bimanual object manipulation. Journal of Experimental Psychology: Human Perception and Performance, 31, 145156.

Lam, M., McFee, K., Chua, R., \& Weeks, D. J. (2006). Macroscopic aspects of gross motor control: A test of the end-state comfort effect. Research Quarterly for Exercise and Sport, 77, 396-400.

Land, M. F. \& Hayhoe, M. (2001). In what ways do eye movements contribute to everyday activities? Vision Research, 41, 3559-3565.

Levin, I. P., Schneider, S. L., \& Gaeth, G. J. (1998). All frames are not created equal: A typology and critical analysis of framing effects. Organizational behavior and human decision processes, 76, 149-188.

Marteniuk, R. G., MacKenzie, C. L., \& Baba, D. M. (1984). Bimanual movement control: Information processing and interaction effects. Quarterly Journal of Experimental Psychology, 36, 335-365.

Mechsner, F., Kerzel, D., Knoblich, G. \& Prinz, W. (2001). Perceptual basis of bimanual coordination. Nature, 414, 69-73.

Mechsner, F., \& Knoblich, G. (2004). Do muscles matter for coordinated action?. Journal of Experimental Psychology: Human Perception and Performance, 30, 490. 
Murchison, N. M., \& Proctor, R. W. (2013). Spatial compatibility effects with unimanual and bimanual wheel-rotation responses: An homage to Guiard (1983). Journal of Motor Behavior, 45, 441-454.

Oldfield, R.C. (1971). The assessment and analysis of handedness: The Edinburgh inventory. Neuropsychologia, 9, 97-113.

Oliveira, F. \& Ivry, R. B. (2008).The representation of action: Insights from bimanual coordination. Current Directions In Psychological Science, 17, 130-135.

Piaget, J. (1954). The construction of reality in the child. New York: Basic Books.

Proctor, R. W. \& Reeve, G. (Eds.) (1990). Stimulus-response compatibility. Amsterdam: North-Holland.

Proctor, R. W., \& Vu, K.-P. L. (2006). Stimulus-response compatibility principles: Data, theory, and application. Boca Raton, FL: CRC Press.

Rosenbaum, D. A., Carlson, R. A. \& Gilmore, R. O. (2001) Acquisition of intellectual and perceptual-motor skills. Annual Review of Psychology, 52, 453-470.

Rosenbaum, D. A., Chapman, K. M., Weigelt, M., Weiss, D. J., \& van der Wel, R. (2012). Cognition, action, and object manipulation. Psychological Bulletin, 138, 924946.

Rosenbaum, D. A., Herbort, O., van der Wel, R., \& Weiss, D. J. (2014). What's in a grasp? American Scientist, 102, 366-373.

Rosenbaum, D. A., Marchak, F., Barnes, H. J., Vaughan, J., Slotta, J., \& Jorgensen, M. (1990). Constraints for action selection: Overhand versus underhand grips. In M. Jeannerod (Ed.), Attention and Performance XIII: Motor representation and control (pp. 321-342). Hillsdale, NJ: Lawrence Erlbaum Associates. 
Schmidt, R. A. \& Bjork, R. A. (1992). New conceptualizations of practice: Common principles in three paradigms suggest new concepts for training. Psychological Science, 3, 207-214.

Tversky, A., Kahneman, D. (1986). Rational Choice and the Framing of Decisions. The Journal of Business, 59, S251-S278.

van der Wel, R. P. Fleckenstein, R., Jax, S., \& Rosenbaum, D. A. (2007). Hand path priming in manual obstacle avoidance: Evidence for abstract spatio-temporal forms in human motor control. Journal of Experimental Psychology: Human Perception and Performance, 33, 1117-1126.

Van der Wel, R. P. \& Rosenbaum, D. A. (2010). Bimanual grasp planning reflects changing rather than fixed constraint dominance. Experimental Brain Research, 205, $351-362$.

Weigelt, M., Kunde, W., \& Prinz, W. (2006). End-state comfort in bimanual object manipulation. Experimental Psychology, 53, 143-148.

Weiss, D. J., Wark, J. D., \& Rosenbaum, D. A. (2007). Monkey see, monkey plan, monkey do: The end-state comfort effect in cotton-top tamarins (Saguinus Oedipus). Psychological Science, 18, 1063-1068. 